\title{
Formación inicial docente de la carrera Educación Comercial: aportes desde la Educación Superior Universitaria Costarricense
}

\author{
Initial teacher training of the major in Commercial Education: \\ contributions from the Costa Rican Higher University Education
}

\author{
Xinia Corrales-Escalante \\ Académica, Escuela de Secretariado Profesional \\ Universidad Nacional \\ Heredia, Costa Rica \\ xinia.corrales.escalante@una.cr \\ Carolina Hernández-Chaves \\ Académica, Escuela de Secretariado Profesional \\ Universidad Nacional \\ Heredia, Costa Rica \\ carolina.hernandez.chaves@una.cr
}

Recibido - Received: 28 / 02 / 2020

Corregido - Revised: 04 / 05 / 2020

Aceptado - Accepted: 22 / 05 / 2020

DOI: https://doi.org/10.22458/ie.v22i32.2829

URL: https://revistas.uned.ac.cr/index.php/innovaciones

\begin{abstract}
Resumen: En las últimas cinco décadas Costa Rica ha evidenciado un interés especial en la Educación Técnica y la Formación Profesional, especialmente en la modalidad Comercial y Servicios, lo que ha generado la necesidad de establecer acciones estratégicas para responder a los nuevos retos y oportunidades de un mundo cambiante en los ámbitos económico, social, cultural, ambiental y tecnológico. Desde la Escuela de Secretariado Profesional de la Universidad Nacional, se ha dado respuesta a la creciente demanda de profesorado de calidad para ocupar diversos puestos en el Ministerio de Educación, con personas graduadas de la carrera de Educación Comercial, específicamente para impartir las especialidades de Secretariado Ejecutivo, Secretariado Ejecutivo Bilingüe y Ejecutivo para Centros de Servicio en los colegios de las ramas técnicas y académicas.
\end{abstract}

Palabras clave: docencia, educación técnica, enseñanza comercial, enseñanza superior, formación de docentes de educación técnica

Summary: In the last five decades, Costa Rica has shown a special interest in Technical Education and Professional Training, especially in the Commercial and Services modality, which has generated the need to establish strategic actions to respond to the new challenges and opportunities of a changing world in the economic, social, cultural, environmental and technological fields. From the School of Professional Secretariat of the Universidad Nacional, there has been a response to the growing demand for quality teachers to occupy various positions in the Ministry of Education, with graduates from the Commercial Education major. Specifically they will teach the specialties of Executive Secretariat, Executive Bilingual Secretariat and Executive for Service Centers in colleges of technical and academic branches.

Key Words: teaching, technical education, commercial education, higher education, technical education teacher training 
La persona profesional en Educación Comercial que forma la Escuela de Secretariado Profesional de la Universidad Nacional desde junio de 1980 responde a una necesidad de docentes con una formación interdisciplinaria, con habilidades para planificar, realimentar y transformar los procesos educativos con metodologías activas para el aprendizaje en el área comercial y de secretariado en diversas instituciones de educación formal y no formal. Entre estas instituciones se pueden citar: la Universidad Estatal a Distancia, la Universidad Técnica Nacional, la Universidad Nacional y la Universidad Independiente, el Ministerio de Educación Pública, el Instituto Nacional de Aprendizaje y las cinco instituciones de educación superior parauniversitarias.

La investigación se centrará en el Ministerio de Educación Pública como institución empleadora desde 1968 de la mayoría del profesorado egresado y graduado de la carrera de Educación Comercial. El profesorado puede laborar en cinco especialidades pertenecientes a la modalidad Comercial y Servicios de la rama técnica: Ejecutivo para Centros de Servicios, Executive Service Center, Secretariado Ejecutivo, Secretariado Bilingüe y Bilingual Secretary. Asimismo, puede impartir los talleres exploratorios, talleres de orientación tecnológica y las tecnologías de la rama académica de la modalidad Comercial y Servicios.

En la Universidad Nacional, el término egresado hace referencia a la persona estudiante que ha aprobado todos los créditos de la carrera en los diferentes grados, y el graduado es quien posterior a la aprobación de todos los requisitos establecidos realiza el proceso de juramentación correspondiente que lo acredita con el título universitario.

El objetivo general del artículo es analizar los principales aportes que ha brindado la Escuela de Secretariado Profesional de la Universidad Nacional en la formación inicial del profesorado de la carrera Educación Comercial que labora en el Ministerio de Educación Pública. Para el logro del objetivo general se plantean los siguientes objetivos específicos: a) Identificar los espacios laborales en el Ministerio de Educación Pública en los que pueden ejercer las personas egresadas o graduadas de la carrera Educación Comercial de la Universidad Nacional, b) Comparar la congruencia existente entre las capacidades y competencias de las personas graduadas o egresadas de la carrera Educación Comercial de la Universidad Nacional para satisfacer las demandas laborales del Ministerio de Educación Pública, c) Analizar los aspectos que deben incluirse en la formación inicial de las personas graduadas o egresadas de la carrera Educación Comercial de la Universidad Nacional para lograr un mejor desempeño en el Ministerio de Educación Pública.

La investigación refleja resultados asociados con la necesidad de que la Escuela de Secretariado Profesional mantenga un vínculo con el Ministerio de Educación Pública para que el perfil de las personas profesionales que se gradúan o egresan de la carrera de Educación Comercial sea congruente con el perfil de la persona educadora que requiere contratar el Ministerio de Educación Pública.

\section{REFERENTES HISTÓRICOS INTERNACIONALES Y NACIONALES DEL SISTEMA EDUCATIVO COSTARRICENSE}

El Sistema Educativo Costarricense cimienta su fundamento jurídico en las disposiciones internacionales relativas al derecho a la educación, establecidas en la Declaración Universal de Derechos Humanos (1948) y en la adhesión de Costa Rica a la Organización de las Naciones Unidas para la Educación, la Ciencia y la Cultura, mejor conocida como la UNESCO (1949). 
A nivel nacional, el principal referente jurídico es la Constitución Política de 1949, la cual destina el título VII, artículos del 76 al 89, a la educación y a la cultura. En esos artículos se establece que la educación es un proceso integral correlacionado desde preescolar hasta la universidad, asimismo, indica la obligatoriedad y gratuidad desde preescolar hasta educación diversificada, así como, la obligación del Estado de proveer de recursos pecuniarios a quienes carezcan de medios económicos para continuar con sus estudios.

En materia económica, indica el porcentaje del gasto público destinado a la educación estatal, incluida la superior y que el Estado dotará de rentas propias a las universidades estatales, independientemente de las originadas en estas instituciones. Y, por último, señala la libertad de cátedra como principio fundamental de la enseñanza universitaria.

Asimismo, la Ley Fundamental de Educación de 1957, establece los fines de la educación costarricense y define la estructura del sistema educativo en Educación Preescolar, General Básica, Diversificada y Superior.

\section{ENSEÑANZA DEL SECRETARIADO EN EL MINISTERIO DE EDUCACIÓN PÚBLICA}

Desde sus inicios en 1968, la enseñanza del secretariado en el Ministerio de Educación Pública ha sido parte de la formación del estudiantado de educación técnica. La educación técnica y profesional como parte del Sistema Educativo Costarricense surge en 1953, con la creación del Colegio Vocacional de Artes y Oficios de Cartago (COVAO) y el Colegio Vocacional Monseñor Sanabria, donde el estudiantado sin recursos económicos y sin oportunidades de ingreso a la educación superior se formaba profesional y moralmente.

La educación vocacional inicia de manera oficial en el país en 1956, con la aprobación del primer plan de estudios específico para esta modalidad. Además, se establece el financiamiento estatal para la educación técnica y profesional, así como su universalización.

Más de una década después de la oficialización de la educación técnica y vocacional, el Consejo Superior de Educación aprueba en 1968 el Acta 107-68, el Plan de Estudios para el Segundo Ciclo, Sección Profesional, Modalidad Secretariado, para cuarto, quinto y sexto año. El Plan incluía las siguientes materias académicas: Español, Estudios Sociales, Ciencias, Matemáticas, Inglés y Ética. Respecto, a las materias profesionales, incluía: Mecanografía, Taquigrafía, Lenguaje y Correspondencia Comercial, Archivo, Técnicas Secretariales, Organización y Administración de Oficinas, Documentación Comercial, Desarrollo de la Personalidad, Principios de Contabilidad General y Elementos de Estadística.

Igualmente, en esa acta se aprueba el Plan de Estudios para las carreras de Oficinista para Primero y Segundo año. Las materias académicas para oficinista eran: Español (redacción comercial), Taquigrafía, Mecanografía, Desarrollo de la Personalidad, Prácticas de Oficina, Archivo, Organización y Administración de Oficinas y Matemáticas Comerciales. En el caso de auxiliar de oficina, Primer año, el estudiantado tenía que cursar: Español (gramática, redacción, ortografía, lectura), Prácticas de Oficina, Matemáticas Comerciales, Mecanografía, Desarrollo de la Personalidad, Documentación Comercial y Archivo.

Tres décadas después, en 1992 fue aprobada por el Consejo Superior de Educación la Oferta Educativa de la Educación Técnica que fue modificada en el año 1995 y actualizada en el año 2003. La oferta educativa todavía se encuentra vigente y se caracteriza por ser:

[...] parte integrante de la educación general que se imparte en las instituciones de educación secundaria formal, un medio de acceso a los sectores profesionales (agropecuario, industrial, comercial y servicios) que contribuye a crear nuevas oportunidades de movilidad social, parte de la educación continua. (MEP, p. 14, 2003) 
La Educación Técnica es un sub-sistema del Sistema Educativo, que incluye en el III Ciclo de la Educación General Básica la exploración vocacional y en la Educación Diversificada el técnico en el nivel medio. A su vez, se divide en tres modalidades: Comercial y Servicios, Industrial y Agropecuaria. Dentro de la modalidad Comercial y Servicios se ofrece las especialidades: Secretariado Bilingüe, Secretariado Ejecutivo y Ejecutivo para Centros de Servicios. Para dar sustento a estas especialidades, fueron diseñados los programas de estudio:

- Bilingual Secretary. Niveles décimo, undécimo y duodécimo año.

- Secretariado Ejecutivo. Niveles décimo, undécimo y duodécimo año.

- Ejecutivo para Centros de Servicio. Niveles décimo, undécimo y duodécimo año.

- Executive Call Centers. Niveles décimo, undécimo y duodécimo año.

Las personas graduadas de los colegios técnicos profesionales reciben el título de Técnico de Nivel Medio en la especialidad cursada. La rama técnica se diferencia de las ramas académicas y artísticas, en que el estudiantado debe cursar un año adicional de formación específica.

\section{FORMACIÓN INICIAL DOCENTE EN EDUCACIÓN COMERCIAL EN LA UNIVERSIDAD NACIONAL}

La apertura de la Escuela de Secretariado Profesional (ESP) de la Universidad Nacional, marcó un hito en la historia de la educación comercial a nivel universitario en Costa Rica porque fue la primera universidad estatal en impartir la carrera, status quo que todavía mantiene por ser la única. La carrera fue aprobada por la Comisión Organizadora AD-HOC en la Sesión N 82, celebrada el 13 de febrero de 1974, según consta en el Folio 00189 del acta correspondiente y que a la letra dice:

\section{ARTÍCULO XI APERTURA DE LA ESCUELA DE SECRETARIADO PROFESIONAL}

Se ACUERDA declarar oficialmente inaugurada la Escuela de Secretariado Profesional, adscrita a la Facultad de Ciencias Sociales de la Universidad Nacional. ACUERDO FIRME.

La carrera Educación Comercial como se denomina actualmente ha sufrido transformaciones en su oferta de estudio, ya que inició un 26 de junio de 1980 con un plan de estudios llamado Bachillerato en Secretariado Profesional con énfasis en Educación. En 1982 se gradúa la primera generación de estudiantes. Desde 1982 hasta el 2019 se cuenta con 37 cohortes de personas graduadas, para un total de 850 estudiantes con títulos distribuidos en diplomado, bachillerato y licenciatura.

El Plan de Estudios de las carreras Licenciatura en Educación Comercial y Bachillerato en Educación Comercial con salida lateral al Diplomado en Educación Comercial, es compartido entre la Escuela de Secretariado Profesional donde se imparten los contenidos de la especialidad y la División de Educología, unidad académica del Centro de Investigación y Docencia en Educación (CIDE), que imparte el componente pedagógico.

La educación comercial se concibe como la formación integral de las personas docentes que preparan al profesional en el área secretarial y la gestión comercial y administrativa. Esta educación está vinculada con las siguientes áreas: administración y gestión en la oficina, comunicación en español e inglés; técnicas secretariales, tecnología y pedagogía. Complementariamente se ofrece formación en: psicopedagogía, derecho, psicología, filosofía, sociología y además se desarrolla la vocación investigadora en el estudiantado, para fortalecer los procesos y los vincule con la realidad educativa en la que trabajará. 
Asimismo, se ofrece al estudiantado diversos proyectos, programas y actividades de extensión, investigación y docencia en los cuales puede involucrarse de manera voluntaria o como requisito de algún curso.

\section{ESCUELA DE SECRETARIADO PROFESIONAL}

- Actividad permanente en procesos prácticos para la Formación Docente en la Carrera de Educación Comercial.

- Actividad permanente en formación profesional dual en Administración de Oficinas y Educación Comercial.

- Actividad permanente de actualización y desarrollo profesional en Administración de Oficinas y Educación Comercial. Su objetivo es organizar un congreso al año, alternando entre Administración de Oficinas y Educación Comercial.

- Revista rESPaldo. Revista Internacional de Administración de Oficinas y Educación Comercial para promover y divulgar la producción intelectual de la comunidad académica científica nacional e internacional en el campo de la administración de oficinas y la educación comercial.

\section{EDUCOLOGÍA}

- Fortalecimiento de la mediación pedagógica de los graduados en diversas carreras de enseñanza secundaria. Su objetivo es desarrollar acciones formativas y de seguimiento de las personas graduadas que permitan el mejoramiento de la mediación pedagógica de los profesionales en las carreras de la enseñanza secundaria.

Además, entre los aportes innovadores que se ofrece al estudiantado de la carrera de Educación Comercial se puede señalar el uso del aula virtual institucional como apoyo a los procesos de aprendizaje, la integración de la virtualidad en los cursos universitarios propiciando cursos bimodales (presencialvirtual) con estrategias didácticas orientadas a la ejecución de los contenidos de los cursos mediante una metodología innovadora para lograr el aprendizaje del estudiantado de una manera integral.

Un logro de la Escuela de Secretariado Profesional para el estudiantado de Educación Comercial fue la acreditación de la carrera por medio del Sistema Nacional de Acreditación de la Educación Superior (SINAES) en el año 2018, lo que garantiza al estudiante y al ente empleador la calidad de la carrera.

Las personas graduadas o egresadas del plan de estudios están facultadas para impartir las diferentes especialidades y se ubican en las siguientes categorías profesionales de acuerdo con el Manual Descriptivo de Especialidades Docentes de la Dirección General de Servicio Civil. 
TABLA 1

Especialidades y grupos profesionales

\begin{tabular}{|c|c|c|c|c|}
\hline Especialidad & Sub-áreas & Título & Rango de aplicación & $\begin{array}{l}\text { Grupo } \\
\text { profesional }\end{array}$ \\
\hline \multirow{3}{*}{$\begin{array}{l}\text { Secretariado } \\
\text { Ejecutivo } \\
\text { código } 076000\end{array}$} & \multirow{3}{*}{$\begin{array}{l}\text { Gestión Empresarial, } \\
\text { Destrezas Computacionales, } \\
\text { Comunicación Empresarial, } \\
\text { Administración de } \\
\text { Documentos. }\end{array}$} & $\begin{array}{l}\text { Licenciatura en Educación Comercial } \\
\text { con énfasis en Docencia, }\end{array}$ & $\begin{array}{l}\text { Profesor de enseñanza } \\
\text { técnico profesional }\end{array}$ & VT6 \\
\hline & & $\begin{array}{l}\text { Bachillerato en Educación Comercial } \\
\text { con énfasis en Docencia, }\end{array}$ & & VT5 \\
\hline & & $\begin{array}{l}\text { Diplomado en Educación Comercial con } \\
\text { énfasis en Docencia. }\end{array}$ & & VT2 \\
\hline \multirow{3}{*}{$\begin{array}{l}\text { Ejecutivo para } \\
\text { Centros de Servicio } \\
\text { código } 035000\end{array}$} & \multirow{3}{*}{$\begin{array}{l}\text { Gestión Empresarial, } \\
\text { Destrezas Computacionales, } \\
\text { Comunicación Empresarial. }\end{array}$} & $\begin{array}{l}\text { Licenciatura en Educación Comercial } \\
\text { con énfasis en Docencia, }\end{array}$ & $\begin{array}{l}\text { Profesor de enseñanza } \\
\text { técnico profesional }\end{array}$ & VT6 \\
\hline & & $\begin{array}{l}\text { Bachillerato en Educación Comercial } \\
\text { con énfasis en Docencia, }\end{array}$ & & VT5 \\
\hline & & $\begin{array}{l}\text { Diplomado en Educación Comercial con } \\
\text { énfasis en Docencia. }\end{array}$ & & VT2 \\
\hline \multirow{2}{*}{$\begin{array}{l}\text { Executive Secretarial } \\
\text { Management } \\
\text { código } 107000\end{array}$} & \multirow{2}{*}{$\begin{array}{l}\text { Gestión Empresarial, } \\
\text { Cultura de la Calidad, } \\
\text { Salud Ocupacional, } \\
\text { Tecnologías de Información } \\
\text { Aplicadas a la Especialidad. }\end{array}$} & $\begin{array}{l}\text { Licenciatura en Secretariado Profesional } \\
\text { con concentración en Educación } \\
\text { Comercial, }\end{array}$ & $\begin{array}{l}\text { Profesor de enseñanza } \\
\text { técnico profesional } \\
\text { bilingüe }\end{array}$ & VT6 \\
\hline & & $\begin{array}{l}\text { Profesorado de Estado en Educación } \\
\text { Comercial con énfasis Secretariado }\end{array}$ & & VT4 \\
\hline $\begin{array}{l}\text { Secretariado } \\
\text { Generalista } \\
\text { código } 095000\end{array}$ & No aplica & $\begin{array}{l}\text { ídem Secretariado Ejecutivo y Ejecutivo } \\
\text { para Centros de Servicios }\end{array}$ & $\begin{array}{l}\text { Asesor nacional (G. } \\
\text { de E.) }\end{array}$ & VT6 \\
\hline
\end{tabular}

Fuente: Elaboración propia con elementos del Manual Descriptivo de Especialidades Docentes de la Dirección General de Servicio Civil (2019).

En la tabla 1 se observa que para ejercer en todas las especialidades es necesario tener un título en la carrera de Educación Comercial.

Es importante mencionar que las personas docentes que tengan la atinencia para la especialidad Executive Secretarial Management pueden impartir los programas de estudio Bilingual Secretary y Executive Service Centers.

Se aclara que en la especialidad Secretariado Ejecutivo se menciona que el programa de estudios incluye la subárea Administración de Documentos. No obstante, en el programa de estudios se indica que el nombre correcto de la subárea es Comunicación Empresarial y Administración de Documentos.

Además, se observa que en este Manual se denomina a la especialidad Ejecutivo para Centros de Servicio, sin embargo, el programa de estudio tiene como nombre Ejecutivo para Centros de Servicios. Tampoco se visualiza la subárea Administración para Centros de Servicio que se encuentra incorporada en el programa de estudios de Ejecutivo para Centros de Servicios.

De acuerdo con el Manual, las personas graduadas o egresadas están facultadas para impartir los talleres exploratorios, talleres de orientación tecnológica y las tecnologías, que se describen a continuación. 
TABLA 2

Talleres exploratorios, talleres de orientación tecnológica y tecnologías

Talleres exploratorios

- Técnicas y diseño de recursos para la oficina.

- Oficina moderna.

- Archivar para el futuro.

- Digitación computacional.
Talleres orientación tecnológica

- Técnicas y Diseño de recursos para la oficina.

- Oficina moderna.

- Archivar para el futuro.

- Digitación computacional.

- Aprestamiento para la automatización secretarial.

- Juegos didácticos.

Fuente: Elaboración propia con elementos del Manual Descriptivo de Especialidades Docentes de la Dirección General de Servicio Civil (2019).

\section{MATERIALES Y MÉTODOS}

El estudio que se realizó se fundamentó en un modelo de análisis con un enfoque cuantitativo descriptivo, mediante la medición numérica y estadística que describe las percepciones del grupo de empleadores con respecto a diversos temas relacionados con la carrera Bachillerato y Licenciatura en Educación Comercial, tales como información general, correspondencia con el contexto, plan de estudios y satisfacción del desempeño, entre otros.

\section{Población y muestra}

Para realizar el estudio y garantizar representatividad, la Comisión de Autoevaluación de la Escuela de Secretariado Profesional eligió la población total según la base de datos de personas empleadoras pertenecientes al Ministerio de Educación Pública para el periodo 2014-2017. Se enviaron invitaciones a 23 personas empleadoras, de las cuales 11 personas respondieron el instrumento.

\section{Técnicas de recolección de datos}

La técnica de recolección de datos que se aplicó al grupo de personas empleadoras fue una encuesta elaborada por la Vicerrectoría de Docencia, proceso Seguimiento, Evaluación y Acreditación de Carreras (SEAC) que posteriormente fue revisada, adaptada y validada por la Comisión de Autoevaluación. Las modificaciones finales fueron enviadas al informático de la Facultad de Ciencias Sociales, para incluirlas en la aplicación LimeSurvey. Una vez corregido y editado el instrumento cuestionario en la aplicación, fue nuevamente revisado y validado por la Comisión de Autoevaluación con fines de Acreditación.

El cuestionario que se aplicó estuvo conformado por 15 preguntas y por las siguientes secciones: 
TABLA 3

Datos del instrumento aplicado al grupo de empleadores.

\begin{tabular}{ccc} 
Sección & Nombre & Cantidad preguntas \\
Sección A. & Información general & 4 preguntas \\
Sección B. & Componente correspondencia con el contexto & 4 preguntas \\
Sección C. & Plan de estudios & 6 preguntas \\
Sección D. & Comentarios finales & 1 pregunta \\
\hline
\end{tabular}

Fuente: Comisión Autoevaluación, Informe resultados de opinión, personas empleadoras, 2018.

\section{RESULTADOS}

Para efectos de este artículo solamente se contempló el análisis de los ítems 10 y 13 del instrumento cuestionario con las siguientes dos preguntas, relacionadas con la Sección C. Plan de estudios.

- Valoración de la congruencia existente entre las capacidades y competencias de las personas graduadas de la carrera Bachillerato y Licenciatura en Educación Comercial de la UNA y las demandas de la organización, 2017 (pregunta 10 del cuestionario).

- Valoración de los aspectos que deberían incluir en la formación de la persona graduada de la carrera Bachillerato y Licenciatura en Educación Comercial de la UNA, para lograr un mejor desempeño en esta institución (pregunta 13 del cuestionario).

\section{TABLA 4}

Opinión de la congruencia existente entre las capacidades y competencias de las personas graduadas y las demandas de la organización, 2017. Bachillerato en Educación Comercial

\begin{tabular}{|c|c|c|c|c|c|c|}
\hline \multirow{2}{*}{ Conocimientos } & \multicolumn{2}{|c|}{ Poco congruente } & \multicolumn{2}{|c|}{ Congruente } & \multicolumn{2}{|c|}{ Muy congruente } \\
\hline & Abs. & $\%$ & Abs. & $\%$ & Abs. & $\%$ \\
\hline El currículo de la Educación Comercial y su proceso & 0 & 0,00 & 5 & 45,45 & 6 & 54,55 \\
\hline Los sistemas de evaluación utilizados en la Educación Comercial & 0 & 0,00 & 5 & 45,45 & 6 & 54,55 \\
\hline $\begin{array}{l}\text { Metodologías, estrategias y materiales didácticos, específicos } \\
\text { para el desarrollo del proceso de enseñanza y aprendizaje de la } \\
\text { Educación Comercial }\end{array}$ & 0 & 0,00 & 5 & 45,45 & 6 & 54,55 \\
\hline
\end{tabular}

Fuente: Comisión de Autoevaluación con datos del instrumento percepción grupo de empleadores.

TABLA 5

Opinión de la congruencia existente entre las capacidades y competencias de las personas graduadas y las demandas de la organización en relación con el Bachillerato en Educación Comercial, 2017.

\begin{tabular}{|c|c|c|c|c|c|c|}
\hline \multirow{2}{*}{ Habilidades } & \multicolumn{2}{|c|}{ Poco congruente } & \multicolumn{2}{|c|}{ Congruente } & \multicolumn{2}{|c|}{ Muy congruente } \\
\hline & Abs. & $\%$ & Abs. & $\%$ & Abs. & $\%$ \\
\hline Analiza el fenómeno educativo multidisciplinariamente. & 1 & 9,09 & 2 & 18,18 & 8 & 72,73 \\
\hline $\begin{array}{l}\text { Expresa argumentos de manera coherente y clara, } \\
\text { tanto en forma oral como escrita. }\end{array}$ & 0 & 0,00 & 4 & 36,36 & 7 & 63,64 \\
\hline $\begin{array}{l}\text { Planifica el proceso de enseñanza y aprendizaje } \\
\text { en Educación Comercial }\end{array}$ & 0 & 0,00 & 3 & 27,27 & 8 & 72,73 \\
\hline $\begin{array}{l}\text { Contribuye a la transformación y mejoramiento } \\
\text { de la realidad en el campo de la Educación Comercial. }\end{array}$ & 0 & 0,00 & 4 & 36,36 & 7 & 63,64 \\
\hline
\end{tabular}

Fuente: Comisión de Autoevaluación con datos del instrumento percepción grupo de empleadores. 
TABLA 6

Opinión de la congruencia existente entre las capacidades y competencias de las personas graduadas y las demandas de la organización en relación con el Bachillerato en Educación Comercial, 2017.

\begin{tabular}{|c|c|c|c|c|c|c|}
\hline \multirow{2}{*}{ Actitudes } & \multicolumn{2}{|c|}{ Poco congruente } & \multicolumn{2}{|c|}{ Congruente } & \multicolumn{2}{|c|}{ Muy congruente } \\
\hline & Abs. & $\%$ & Abs. & $\%$ & Abs. & $\%$ \\
\hline $\begin{array}{l}\text { Promueve la comprensión de los valores humanísticos } \\
\text { universales y costarricenses, mediante la reflexión crítica } \\
\text { y la aplicación de ellos. }\end{array}$ & 0 & 0,00 & 4 & 36,36 & 7 & 63,64 \\
\hline $\begin{array}{l}\text { Asume la responsabilidad de actualizar sus conocimientos en } \\
\text { forma permanente. }\end{array}$ & 1 & 9,09 & 4 & 36,36 & 6 & 54,55 \\
\hline $\begin{array}{l}\text { Mantiene una actitud de respeto por las diferencias culturales, } \\
\text { religiosas, ideológicas y políticas. }\end{array}$ & 0 & 0,00 & 4 & 36,36 & 7 & 63,64 \\
\hline
\end{tabular}

Fuente: Comisión de Autoevaluación con datos del instrumento percepción grupo de empleadores.

En las tablas 4, 5 y 6 se resumen las opiniones del grupo de personas empleadoras con respecto a la congruencia existente entre las capacidades y competencias de las personas graduadas y las demandas de la organización a nivel de bachillerato.

Según la tabla 4, 54,55\% de las personas encuestadas consideraron muy congruente el currículo de la Educación Comercial y su proceso con los sistemas de evaluación utilizados, así como las metodologías, estrategias y materiales didácticos, específicos para el desarrollo del proceso de enseñanza y aprendizaje de la Educación Comercial y, mientras $45,45 \%$ lo señalaron congruente.

En la tabla 5, respecto a las habilidades, para la relacionada con analiza el fenómeno educativo multidisciplinariamente, $72,73 \%$ manifestó que es muy congruente, de forma similar para la habilidad planificación del proceso de enseñanza y aprendizaje en Educación Comercial.

Para la habilidad expresa argumentos de manera coherente y clara, tanto en forma oral como escrita, 63,64\% manifestó muy congruente, así como para la habilidad contribuye a la transformación y mejoramiento de la realidad en el campo de la Educación Comercial, ambas habilidades también fueron valoradas por $36,36 \%$ como congruente.

En la tabla 6, respecto a las actitudes, 63,64\% de las personas empleadoras consideraron como muy congruente las siguientes: promueve la comprensión de los valores humanísticos universales y costarricenses, mediante la reflexión crítica y la aplicación de ellos, igualmente señalaron que mantienen una actitud de respeto por las diferencias culturales, religiosas, ideológicas y políticas. Las anteriores fueron valoradas por $36,36 \%$ como congruentes.

TABLA 7

Opinión de la congruencia existente entre las capacidades y competencias de las personas graduadas y las demandas de la organización,

Licenciatura en Educación Comercial, 2017.

\section{Conocimientos}

Metodologías de investigación cuantitativa y cualitativa para el desarrollo de investigaciones en el ámbito de la Educación Comercial.

Manejo en grado superior del inglés con propósitos específicos para la enseñanza del área secretarial.

Manejo de herramientas computacionales que posibilite un mejor desempeño en el proceso enseñanza-aprendizaje.

\begin{tabular}{cccccc}
\multicolumn{2}{c}{$\begin{array}{c}\text { Poco congruente } \\
\text { Abs. }\end{array} \quad \%$} & \multicolumn{2}{c}{ Congruente } & \multicolumn{2}{c}{ Muy congruente } \\
1 & 9,09 & 3 & 27,27 & 7 & 63,64 \\
2 & 18,18 & 3 & 27,27 & 6 & 54,55 \\
1 & 9,09 & 2 & 18,18 & 8 & 72,73 \\
\hline
\end{tabular}

Fuente: Comisión de Autoevaluación con datos del instrumento percepción grupo de empleadores. 
TABLA 8

Opinión de la congruencia existente entre las capacidades y competencias de las personas graduadas y las demandas de la organización, Licenciatura en Educación Comercial 2017.

\section{Habilidades}

Aplica conocimientos metodológicos y psicológicos en el proceso de enseñanza y aprendizaje.

Se expresa con fluidez, en forma oral y escrita, en español e inglés. Integra conocimientos teórico-prácticos, con los campos de las ciencias, la educación y el comercio.

Actúa consecuentemente con los valores democráticos de respeto a la diversidad, a la vida en todas sus formas y al derecho a la felicidad personal y colectiva.

\begin{tabular}{cccccc}
\multicolumn{2}{c}{ Poco congruente } & \multicolumn{2}{c}{ Congruente } & \multicolumn{2}{c}{ Muy congruente } \\
Abs. & $\%$ & Abs. & $\%$ & Abs. & $\%$ \\
0 & 0,00 & 5 & 45,45 & 6 & 54,55 \\
3 & 27,27 & 2 & 18,18 & 6 & 54,55 \\
0 & 0,00 & 5 & 45,45 & 6 & 54,55 \\
0 & 0,00 & 5 & 45,45 & 6 & 54,55 \\
\hline
\end{tabular}

Fuente: Comisión de Autoevaluación con datos del instrumento percepción grupo de empleadores.

TABLA 9

Opinión de la congruencia existente entre las capacidades y competencias de las personas graduadas y las demandas de la organización, Licenciatura en Educación Comercial, 2017.

\section{Actitudes}

Manifiesta compromiso ético y moral en el desempeño de sus funciones como docente en el campo de la Educación Comercial.

Cultiva un interés permanente por la investigación en su área profesional.

Demuestra compromiso frente a los problemas educativos y sociales del país.

Demuestra una actitud crítica de las innovaciones metodológicas y técnicas para adecuarlas a las necesidades del entorno.

\begin{tabular}{cccccc}
\multicolumn{2}{c}{$\begin{array}{c}\text { Poco congruente } \\
\text { Abs. }\end{array}$} & \multicolumn{2}{c}{ Congruente } & \multicolumn{2}{c}{ Muy congruente } \\
0 & 0,00 & 3 & 27,27 & 8 & 72,73 \\
1 & 9,09 & 3 & 27,27 & 7 & 63,64 \\
0 & 0,00 & 4 & 36,36 & 7 & 63,64 \\
1 & 9,09 & 2 & 18,18 & 8 & 72,73
\end{tabular}

Fuente: Comisión de Autoevaluación con datos del instrumento percepción grupo de empleadores.

En las tablas 7, 8 y 9 se exponen las opiniones de las personas empleadores con respecto a la congruencia existente entre las capacidades y competencias de las personas graduadas y las demandas de la organización.

De las tablas 7 y 8 se desprende que el conocimiento de herramientas computacionales posibilita un mejor desempeño en el proceso enseñanza-aprendizaje, al respecto, $72,73 \%$ opinó muy congruente. También $63,64 \%$ consideró muy congruente las metodologías de investigación cuantitativa y cualitativa para el desarrollo de investigaciones en el ámbito de la Educación Comercial. Por otra parte, 54,55\% considera muy congruente el manejo en grado superior del inglés con propósitos específicos para la enseñanza del área secretarial.

Con respecto a las habilidades se obtuvo lo siguiente: aplica conocimientos metodológicos y psicológicos en el proceso de enseñanza y aprendizaje, 54,55\% lo valoró muy congruente así como también se expresa con fluidez en forma oral y escrita en español e inglés, integra conocimientos teórico-prácticos en los campos de las ciencias, la educación y el comercio, así como actúa consecuentemente con los valores democráticos de respeto a la diversidad, a la vida en todas sus formas y al derecho a la felicidad personal y colectiva. 
En la tabla 9, respecto a las actitudes se expresó: para la actitud manifiesta compromiso ético y moral en el desempeño de sus funciones como docente en el campo de la Educación Comercial, las personas empleadoras manifestaron que es muy congruente $(72,73 \%$ ) y congruente un $27,27 \%$.

Asimismo, en la tabla 9, respecto a que demuestra una actitud crítica de las innovaciones metodológicas y técnicas para adecuarlas a las necesidades del entorno, $72,73 \%$ lo manifestó como muy congruente y $18,18 \%$ como congruente.

En lo que se refiere a demuestra compromiso frente a los problemas educativos y sociales del país, $64,63 \%$ lo considera como muy congruente, mientras lo estima congruente $36,36 \%$. En cuanto a la actitud cultiva un interés permanente por la investigación en su área profesional, 64,63\% lo consideró muy congruente y $27,27 \%$ como congruente.

Respecto a la satisfacción en diferentes campos, se desglosan las respuestas para cada una de las consultas.

C.3. Satisfacción con el desempeño de las personas graduadas de la carrera Bachillerato y Licenciatura en Educación Comercial de la UNA que laboran en esta institución educativa u organización.

El 100\% (11 empleadores) contestaron que sí están satisfechos con el desempeño de las personas graduadas.

C.4. Satisfacción con el perfil de salida de las personas graduadas de la carrera Bachillerato y Licenciatura en Educación Comercial de la UNA que laboran en esta organización,

El 100\% (11 personas empleadoras) contestaron que sí están satisfechos con el perfil de las personas graduadas.

C.5. Valoración de los aspectos que deberían incluir en la formación de la persona graduada de la carrera Bachillerato y Licenciatura en Educación Comercial de la UNA, para lograr un mejor desempeño en esta empresa o institución.

La totalidad de las personas empleadoras (11) contestó lo siguiente:

1. “Deberían tener bastante práctica en la empresa privada para que los conocimientos que vayan a impartir no sean solo teóricos sino también vivencias personales, experiencias reales para tener una base sólida para incentivar a sus alumnos a escoger esta carrera". E01-CBLEC.

2. "Incluir de mejor manera el tema de herramientas tecnológicas y paquetes computacionales relacionados directamente con la carrera". E02-CBLEC.

3. “Formación en valores, técnicas y metodologías de enseñanza y aprendizaje e integración de las TIC a los procesos educativos". E03-CBLEC.

4. "Actualización de conocimientos en legislación docente, reglamento carrera profesional, educación técnica, práctica profesional supervisada". E04-CBLEC.

5. “Deben prepararse más en inglés, manejo de software: Microsoft Office sobre todo Access y Registro electrónico". E05-CBLEC.

6. “Por tratarse de una institución de formación en educación media y técnico profesional, el principal aporte de los profesionales graduados de su escuela es referente a la docencia. Considero que este aspecto no es del todo tomado en cuenta pues los profesionales que ustedes gradúan están más orientado [sic] a la atención propia de oficina, sin embargo son ampliamente competentes por su formación". E06-CBLEC. 
7. "Considerar reforzar la parte académica y la práctica real". E07-CBLEC.

8. "Manejo de grupos, manejo del aula Liderazgo". E08-CBLEC.

9. "La funcionaria es excelente". E09-CBLEC.

10. "Importante acotar que se debe reforzar los conocimientos en el área Contable, Matemática Financiera y Estadística para que la persona graduada en esta carrera esté más preparada integralmente, acorde con el Plan de Estudios del Ministerio de Educación. Podría tener más conocimientos en andragogía. También en aspectos de la evaluación de los aprendizajes, tales como confeccionar rúbricas, hojas de cotejo, entre otros". E10-CBLEC.

11. "Seguir reforzando nuevas técnicas y metodologías educativas y ampliar idiomas". E11-CBLEC0.

La siguiente tabla presenta datos estadísticos extraídos del Departamento de Análisis Estadístico del MEP acerca de la cantidad de personas graduadas en las diferentes especialidades.

TABLA 10

Cantidad de personas graduadas en la modalidad Comercial y Servicios Especialidades Secretariados y Ejecutivos para Centros de Servicios. Período 2010-2018.

\begin{tabular}{lrrrrrrrrr}
\multicolumn{1}{c}{ Modalidad y Especialidad } & $\mathbf{2 0 1 0}$ & $\mathbf{2 0 1 1}$ & $\mathbf{2 0 1 2}$ & $\mathbf{2 0 1 3}$ & $\mathbf{2 0 1 4}$ & $\mathbf{2 0 1 5}$ & $\mathbf{2 0 1 6}$ & $\mathbf{2 0 1 7}$ & $\mathbf{2 0 1 8}$ \\
Comercial y Servicios & 3,801 & 4,168 & 3,694 & 4,474 & 5,195 & 6,220 & 6,946 & 7,922 & 9,825 \\
Secretariado Ejecutivo & 969 & 847 & 748 & 892 & 1,015 & 972 & 1,063 & 1,307 & 1,527 \\
Secretariado Bilingue & 72 & 14 & 0 & 0 & 0 & 0 & 0 & 0 \\
$\begin{array}{l}\text { Bilingual Secretary } \\
\text { Secretariado Ejecutivo para Centros }\end{array}$ & 0 & 0 & 31 & 44 & 68 & 62 & 46 & 72 & 120 \\
de Servicios & 307 & 372 & 334 & 0 & 0 & 0 & 0 & 0 \\
$\begin{array}{l}\text { Executive Service Center } \\
\text { Ejecutivos para Centros de Servicios }\end{array}$ & 0 & 0 & 0 & 46 & 53 & 27 & 38 & 33 \\
\hline
\end{tabular}

Fuente: Elaboración propia con datos del Departamento de Análisis Estadístico (MEP, 2019).

De la tabla 10, se infiere que la especialidad con mayor crecimiento es Ejecutivo para Centros de Servicio que aumentó del 11,2\% en el 2013 al 13,5\% en el 2018 y en Bilingual Secretary se observa un leve incremento del $0,8 \%$ en el 2012 al 1,2\% en el 2018. Mientras, que la especialidad Secretariado Ejecutivo disminuyó del 25,5\% en el 2010 al 15,2\% en el 2018 y Executive Service Center se reduce del 1\% en el 2013 al $0,5 \%$ en el 2018. También se observa que las especialidades Secretariado Bilingüe y Secretariado Ejecutivo para Centros de Servicios no registran personas graduadas a partir del 2012 y el 2013, respectivamente.

\section{CONCLUSIONES}

La Escuela de Secretariado Profesional en coordinación con la División de Educología ha evidenciado a lo largo de su historia una formación inicial integral, interdisciplinaria, con un enfoque humanístico, científico, innovador y de calidad de profesionales en Educación Comercial para satisfacer las demandas laborales en las diferentes especialidades de la modalidad Comercial y Servicios del Ministerio de Educación Pública.

Esta unidad académica se ha consolidado en las últimas cuatro décadas como pionera en el campo de la Educación Comercial y única universidad estatal que imparte esta carrera a nivel de diplomado, bachillerato y licenciatura. 
Asimismo, mediante la integración de las áreas sustantivas: docencia, investigación, extensión y producción intelectual atiende y favorece la formación profesional, la búsqueda de nuevo conocimiento científico para mejorar la práctica y aporta a sectores sociales menos favorecidos y excluidos del sistema educativo formal por medio de programas y proyectos especializados. Además, brinda capacitación y actualización a las personas profesionales graduadas y egresadas en servicio.

Las personas graduadas o egresadas de Educación Comercial tienen la atinencia para impartir las modalidades Ejecutivo para Centros de Servicio, Secretariado Ejecutivo, Executive Secretarial Management y Secretariado Generalista, así como los talleres exploratorios, talleres de orientación tecnológica y las tecnologías incluidas en el Manual Descriptivo de Especialidades Docentes de la Dirección General de Servicio Civil (2019).

La carrera Educación Comercial está acreditada por el SINAES lo que garantiza criterios de calidad de la carrera al estudiantado y futuros empleadores.

La oferta educativa de Educación Comercial requiere de personas graduadas con competencias para analizar el fenómeno educativo multidisciplinariamente, tener habilidades para planificar, realimentar y transformar los procesos educativos con metodologías activas para el aprendizaje. Para ello debe desarrollar capacidades investigativas con una actitud crítica hacia las innovaciones curriculares, metodológicas y técnicas.

El profesional en Educación Comercial debe poseer habilidades blandas tales como comprensión de los valores humanísticos universales, respeto por las diferencias culturales, religiosas, ideológicas y políticas, compromiso ético en el desempeño de sus funciones y responsabilidad para promover procesos de aprendizaje centrados en el ser humano.

La Escuela de Secretariado Profesional debe reforzar la enseñanza de metodologías de investigación en el ámbito de la Educación Comercial, aumentar el dominio lingüístico del idioma inglés con propósitos específicos en sus estudiantes, profundizar en los sistemas de evaluación, las metodologías, estrategias y materiales didácticos, específicos para el desarrollo del proceso de aprendizaje de la Educación Comercial.

Desde una perspectiva de la disciplina, las personas graduadas en Educación Comercial deben reforzar los conocimientos en el área contable, matemática financiera y estadística, acorde con los Programas de Estudios del Ministerio de Educación Pública (MEP).

La terminología incluida en el Manual Descriptivo de Especialidades Docentes de la Dirección General de Servicio Civil del 2019 presenta incongruencias con los Programas de Estudios de las diferentes especialidades de la modalidad Comercial y Servicios.

La Escuela de Secretariado Profesional debe mantener un vínculo con el Ministerio de Educación Pública para garantizar que el perfil de las personas profesionales graduadas o egresadas de la carrera Educación Comercial sea congruente con el perfil de la persona educadora que requiere contratar el Ministerio de Educación Pública.

Es importante que la carrera Educación Comercial analice a profundidad los aspectos que deben incluirse en la formación inicial de las personas graduadas o egresadas de la carrera Educación Comercial de la Universidad Nacional para lograr un mejor desempeño en el Ministerio de Educación Pública, lo anterior mediante el rediseño de Plan de Estudios con pertinencia social y laboral. 
Asamblea Legislativa de la República de Costa Rica (1957). Ley N 2160: Ley Fundamental de Educación. San José, Costa Rica. Recuperado de: http://www.pgrweb.go.cr/scij/Busqueda/Normativa/ Normas/nrm_texto_completo.aspx?nValor1=1\&nValor2=31427

Asamblea Nacional Constituyente (1949). Constitución Política de la República de Costa Rica. San José, Costa Rica. Recuperado de: https://www.tse.go.cr/pdf/normativa/constitucion.pdf

Consejo Superior de Educación (1968). Acta n. 107-68. Aprobación del Plan de Estudios Plan de Estudios para el Segundo Ciclo, Sección Profesional, Modalidad Comercial, Especialidad Secretariado. Plan de Estudios para las carreras de Oficinista para el Primer y Segundo Año y Auxiliar de Oficinas para Primer Año. San José, Costa Rica.

Dirección General de Servicio Civil (2019). Manual Descriptivo de Especialidades Docentes. San José, Costa Rica. Recuperado de: http://www.dgsc.go.cr/ts_clase_docente/dgsc_servicios_docente.html

Escuela de Secretariado Profesional (2018). Informe de resultados de opinión de personas empleadoras. Comisión de Autoevaluación Carrera Educación Comercial. (p. 14 a 18).

Estado de la Nación (2012). Cuarto Informe del Estado de la Educación. Informe Final Educación Técnica. San José, Costa Rica. Recuperado de: http://repositorio.conare.ac.cr/bitstream/handle/20.500.12337/841/868.\%20Desempe\%C3\%B10\%20de\%20la\%20educaci\%C3\%B3n\%20general\%20 b\%C3\%A1sica\%20y\%20el\%20ciclo\%20diversificado\%20en\%20Costa\%20Rica_IV\%20Informe\%20 Estado\%20de\%20la\%20Educaci\%C3\%B3n_Libro\%20completo.pdf?sequence=1\&isAllowed=y

Hernández, Fernández y Baptista (2014). Metodología de la Investigación. 5ta Edición: Mc Graw Hill. México D.F. México.

Ministerio de Educación Pública (2003). Oferta Educativa de la Educación Técnica Profesional. Modalidad: Agropecuaria, Industrial, Comercio y Servicios. San José, Costa Rica. Recuperado de: https://mep.janium.net/janium/Documentos/9408.pdf

Ministerio de Educación Pública (2008). Programa de Estudios: Secretariado Ejecutivo. Nivel Décimo, Undécimo y Duodécimo Año. San José, Costa Rica. Recuperado de https://www.mep.go.cr/sites/ default/files/programadeestudio/programas/secretariado-ejecutivo-10.pdf

Ministerio de Educación Pública (2008). Programa de Estudios: Ejecutivo para Centros de Servicios. Nivel Décimo, Undécimo y Duodécimo Año. Recuperado de: https://www.mep.go.cr/sites/default/files/programadeestudio/programas/ejecutivo-centro-servicios-10.pdf

Ministerio de Educación Pública (2011). Programa de Estudios: Bilingual Secretary. Niveles Décimo, Undécimo y Duodécimo Año. San José, Costa Rica. Recuperado de: https://www.mep.go.cr/sites/ default/files/programadeestudio/programas/bilingual-secretary-10.pdf

Ministerio de Educación Pública (2011). Programa de Estudios: Executive Service Center. Nivel Décimo, Undécimo y Duodécimo Año. Recuperado de: https://www.mep.go.cr/sites/default/files/programadeestudio/programas/executive-service-centers-10.pdf

Universidad Nacional. (2004). Plan de Estudios Bachillerato en Educación Comercial con énfasis en Docencia con salida lateral al Diplomado en Educación Comercial y Licenciatura en Educación Comercial con énfasis en Investigación. Heredia, Costa Rica.

Para citar este documento:

Corrales-Escalante, X. y Hernández-Chaves, C. (2020). Formación inicial docente de la carrera Educación Comercial: aportes desde la Educación Superior Universitaria Costarricense. Revista Innovaciones Educativas. 22(32), 91-104. DOI: https://doi.org/10.22458/ie.v22i32.2829 